\title{
Lactobacillus plantarum possesses the capability for wall teichoic acid backbone alditol switching
}

Peter A Bron 1,2,3, Satoru Tomita ${ }^{2,4}$, Iris I van Swam,2, Daniela M Remus ${ }^{1,2,7}$, Marjolein Meijerink ${ }^{1,5,6}$, Michiel Wels ${ }^{1,2}$, Sanae Okada ${ }^{4}$, Jerry M Wells ${ }^{1,5}$ and Michiel Kleerebezem ${ }^{1,2,6,7^{*}}$

\begin{abstract}
Background: Specific strains of Lactobacillus plantarum are marketed as health-promoting probiotics. The role and interplay of cell-wall compounds like wall- and lipo-teichoic acids (WTA and LTA) in bacterial physiology and probiotic-host interactions remain obscure. L. plantarum WCFS1 harbors the genetic potential to switch WTA backbone alditol, providing an opportunity to study the impact of WTA backbone modifications in an isogenic background.

Results: Through genome mining and mutagenesis we constructed derivatives that synthesize alternative WTA variants. The mutants were shown to completely lack WTA, or produce WTA and LTA that lack D-Ala substitution, or ribitol-backbone WTA instead of the wild-type glycerol-containing backbone. DNA micro-array experiments established that the tarlJKL gene cluster is required for the biosynthesis of this alternative WTA backbone, and suggest ribose and arabinose are precursors thereof. Increased tarlJKL expression was not observed in any of our previously performed DNA microarray experiments, nor in qRT-PCR analyses of L. plantarum grown on various carbon sources, leaving the natural conditions leading to WTA backbone alditol switching, if any, to be identified. Human embryonic kidney NF-KB reporter cells expressing Toll like receptor (TLR)-2/6 were exposed to purified WTAs and/or the TA mutants, indicating that WTA is not directly involved in TLR-2/6 signaling, but attenuates this signaling in a backbone independent manner, likely by affecting the release and exposure of immunomodulatory compounds such as LTA. Moreover, human dendritic cells did not secrete any cytokines when purified WTAs were applied, whereas they secreted drastically decreased levels of the pro-inflammatory cytokines IL-12p70 and TNF-a after stimulation with the WTA mutants as compared to the wild-type.

Conclusions: The study presented here correlates structural differences in WTA to their functional characteristics, thereby providing important information aiding to improve our understanding of molecular host-microbe interactions and probiotic functionality.
\end{abstract}

Keywords: Lactobacillus plantarum, Probiotic, Wall teichoic acid, Lipoteichoic acid, tag and tar genes, Immunomodulation

\section{Background}

In Gram-positive bacteria the cytoplasmic membrane is covered by a thick cell wall consisting of multiple layers of peptidoglycan, an essential polymer composed of alternating residues of $\beta$-1-4-linked $N$-acetyl muramic acid (MurNac) and $N$-acetyl-glucosamine (GlcNac) [1]. Several other molecules are present in the cell envelope,

\footnotetext{
* Correspondence: michiel.kleerebezem@nizo.com

${ }^{1}$ TI Food \& Nutrition, Nieuwe Kanaal 9A, 6709PA, Wageningen, The Netherlands

${ }^{2}$ NIZO Food Research, Kernhemseweg 2, 6718ZB, Ede, The Netherlands Full list of author information is available at the end of the article
}

including teichoic acids (TAs), polysaccharides, and extracellular proteins [2-4]. Most Gram-positive bacteria produce two distinct types of TA that make up a substantial percentage of total cell envelop dry-weight; wall teichoic acid (WTA) is covalently anchored to the MurNAC residue of peptidoglycan via a phosphodiester bond, whereas lipoteichoic acid (LTA) is anchored in the cytoplasmic membrane through a glycolipid $[5,6]$. LTAs typically consist of repeating units of glycerol-phosphate [7]. By contrast, WTAs containing a variety of different alditols have been reported [5], although glycerol and ribitol are most common [8].

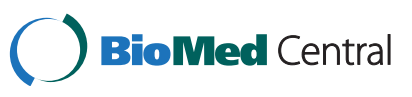


The tag and tar gene cluster products are responsible for WTA biosynthesis of the poly(glycerol-3-phosphate) [poly(Gro-P)] and poly(ribitol-5-phosphate) [poly(Rbo-P)] types, respectively. These genes have been extensively studied in Bacillus subtilis [9] and Staphylococcus aureus $[5,10]$, allowing the identification of most gene functions involved in WTA backbone polymer biosynthesis (Figure 1) and transport, as well as D-Ala and glycosyl substitution of the basic polymer. Initially, construction of tag and tar gene deletion mutants appeared impossible and WTA was thought to be essential $[11,12]$. More recently, WTA deficient mutants were successfully obtained, e.g. in S. aureus [13,14], Lactobacillus plantarum [15], and B. subtilis [16], by deletion of the first gene in the biosynthetic pathway (tagO). This suggests that earlier attempts to disrupt genes which function at a later stage in the pathway result in the accumulation of toxic intermediates or the depletion of components like undecaprenyl phosphate that typically function as a scaffold in cell-wall component biosynthesis [1,17]. Phenotypic analysis of the L. plantarum and B. subtilis tagO mutants indicated an important role for WTA in normal cell elongation and cell-shape maintenance $[15,18]$.

The lactobacilli studied to date have all been shown to produce poly(Gro-P) WTA molecules [17], although some species have been identified that do not produce any WTA, i.e. L. rhamnosus, L. casei, L. fermentum and L. reuteri $[2,3]$. Moreover, L. plantarum strains appear to produce either poly(Gro-P) or poly(Rbo-P) WTA $[3,8]$. Recently, L. plantarum strains, including the most studied strain WCFS1, that encode the tagD1-tagF1-tagF2 genes (tag-locus) were shown to consistently produce poly(Gro-P) WTA, while strains that lack these genes produce poly(Rbo-P) WTA [8]. The production of poly (Rbo-P) WTA in L. plantarum was proposed to depend on the tar locus (tarIJKL) that is universally conserved among L. plantarum strains [8] and was originally annotated as lp_1816-lp_1817-tagB1-tagB2 in the genome sequence of strain WCFS1 [19]. Despite the fact that poly (Gro-P) WTA producing L. plantarum strains consistently

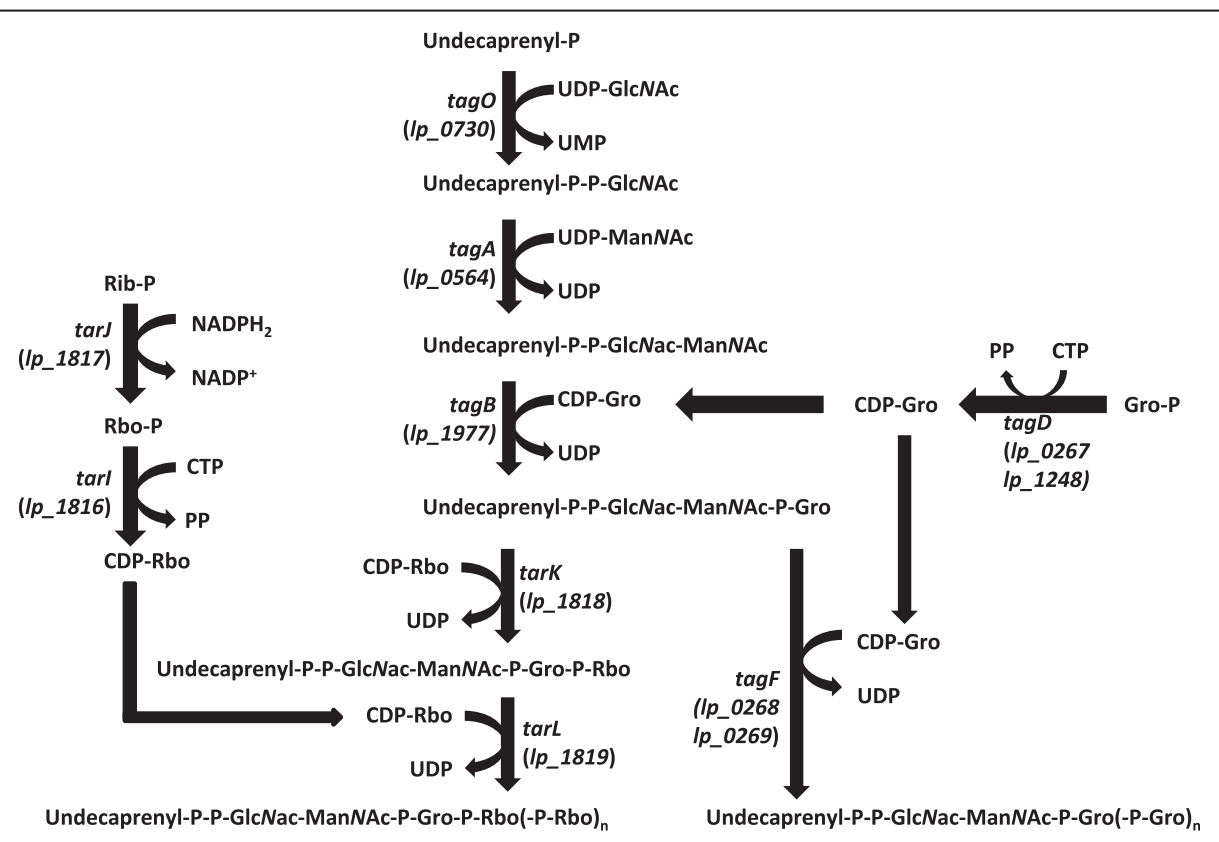

Figure 1 Functions of the proteins encoded by the tag and tar genes in the biosynthesis of poly(Gro-P)- and poly(Rbo-P)-containing WTA backbones, respectively [adapted from [9]. Numbers between brackets indicate the gene-identifiers of tag and tar homologues in the L. plantarum WCFS1 genome and Rib represents ribulose. Regardless of the backbone type, the biosynthesis of WTA is initiated with the consecutive coupling of UDP-activated $\mathrm{N}$-acetylglucosamine (GlcNac) and $\mathrm{N}$-acetylmannosamine (ManNac) to undecaprenyl phosphate on the cytoplasmic side of the cell membrane by TagO and TagA, respectively [5]. Gro-P is CDP-activated by TagD and coupled to this disaccharide linkage unit by the primase TagB. In strains producing poly(Gro-P) WTA the final biosynthetic step involves the coupling of a large but variable amount of CDP-activated Gro-P monomers by the oligomerase TagF. Although TagF homologues (sometimes designated TarF) are present in several strains producing poly(Rbo-P) WTA, these enzymes are generally smaller than their TagF counterparts and their activity is limited to the incorporation of 1 or 2 additional CDP-activated Gro-P monomers [9,62]. In the final biosynthetic steps CDP-activated Rbo-P, produced by TarJ and Tarl, is added to the Gro-P mono-, di- or tri-mer by the primase (TarK) and the oligomerase activity (TarL), resulting in the addition of multiple repeating units of Rbo-P $[5,9,13,63]$. Following its synthesis, the complete WTA polymer is proposed to be transported across the cytoplasmic membrane by the tagGH-encoded ABC transporter, and is subsequently coupled to the 6-hydroxyl of MurNAc in peptidoglycan by an unknown transferase $[5,13]$. These basic WTA polymers can be substituted with D-alanyl esters through the activity of the enzymes encoded in the $d / t$ operon [31,64,65] and/or glycosyl moieties by homologues of TagE or TarM of B. subtilis and S. aureus, respectively [39,40]. 
encode both the tag and tar loci, and therefore possess the genetic capability to synthesize WTA with either backbone alditol, there have been no reports of strains that produce the Rbo-P or both WTA subtypes, indicating that the presence of the tag-locus exclusively dictates WTA production [8].

Following consumption, L. plantarum strains typically survive for several days in the human gastrointestinal tract [20] and adhere to human mucosa [21]. Specific strains of L. plantarum, have demonstrated health-promoting effects on the consumer [22]. For example, consumption of $L$. plantarum strain WCFS1 was shown to trigger mucosal gene expression patterns and cellular pathways that correlate with the establishment of immune tolerance in healthy adults [23]. The same study suggests that the molecular host responses towards L. plantarum WCFS1 in human intestinal mucosa depends on the cell-envelope composition of the culture administered [23]. Moreover, there is compelling evidence that specific Lactobacillus cell-wall components play a pivotal role in the interaction of probiotic lactobacilli with the immune system, e.g. specific tripeptides derived from peptidoglycan modulate immune responses via human NOD-like receptor 1 and 2 (NOD1 and NOD2, [3,24-26]). Furthermore, TLR-2 in association with TLR-1 or TLR-6 plays an important role in the innate immune response by recognizing microbial lipoproteins and lipopeptides [26,27]. To this end, LTA of several Lactobacillus species [28-30], including L. plantarum NCIMB8826 (of which strain WCFS1 is a single colony isolate) [31], was demonstrated to interact with Toll like receptor 2 (TLR-2, $[3,4,26]$ ). Moreover, purified LTA from L. plantarum WCFS1 was shown to elicit the production of the cytokine tumor necrosis factor alpha (TNF- $\alpha$ ) in murine bone-marrow cells in a TLR-2 dependent manner, which is largely dependent on D-Ala substitution of the LTA backbone [31].

In contrast to LTA, the role of WTA in TLR-2-mediated immune signaling is debated [3,32], especially as WTA is not acylated [5] which is considered an essential characteristic for LTA signaling [26]. Moreover, contamination of purified WTA fractions with other immunomodulating bacterial components may have blurred the assignment of the reported effects to WTA-mediated signaling. For example the WTA of $L$. casei Shirota has been proposed to act synergistically with LTA to induce IL-10 production through a TLR-2-dependent pathway [33], while genome comparisons of genetic loci involved in WTA biosynthesis suggest that $L$. casei strains cannot produce WTA [2]. Here, we aimed to clarify the role of WTA by addressing the following questions; is L. plantarum WCFS1 capable of switching its WTA backbone alditol? Can the immune system recognize $L$. plantarum poly(Gro-P) and/or poly (Rbo-P) WTA in addition to LTA?
The fact that some $L$. plantarum strains possess the genetic capacity to produce both WTA-backbone types, offers the possibility to study the physiological consequences and host-signaling induced by these WTA variants in an isogenic background. Therefore, we biochemically analyzed the modified WTA synthesized by L. plantarum WCFS1 mutants demonstrating that (i) a tagO deletion mutant is unable to produce WTA, (ii) a tagF1-2 deletion mutant produces WTA with a poly (Rbo-P) backbone instead of poly(Gro-P), and (iii) a dlt X-D deletion mutant lacks D-Ala substitution of both LTA and WTA. Subsequently, DNA microarrays revealed the consequences of these modifications on bacterial physiology and the WTA biosynthetic pathways. To gain insight into the potential role of WTA and its modifications on host-immune interactions, the differential effect of the mutants and purified WTA derived from these mutants on cytokine production by human monocyte derived dendritic cells, as well as their innate signaling capacity via TLR-2/6 in a NF- $\mathrm{kB}$ reporter cell line, was investigated.

\section{Results}

in silico analysis of the WTA biosynthetic pathways in $L$. plantarum

The NCBI database (http://www.ncbi.nlm.nih.gov/) contains the complete and annotated genome sequences of the L. plantarum strains WCFS1 [19,34] and JDM-1 [35]. By using blastn [36], it was established that both genomes contain homologues of $\operatorname{tag} O, \operatorname{tag} A, \operatorname{tag} D, \operatorname{tag} B$, tarIJKL (tar-locus), and tagGH (Figures 1 and 2A). In contrast to B. subtilis [9] and S. aureus [10], the L. plantarum genes required for biosynthesis and transport of WTA backbones are scattered over 6 chromosomal loci, with a conserved chromosomal organization in L. plantarum WCFS1 and JDM-1. Only the WCFS1 genome contains the additional tag-locus (tagD1-F1-F2) (Figure 2A and $\mathrm{B})$ that was previously predicted to dictate the production of poly(Gro-P) WTA in this strain, while the absence of this locus in strain JDM1 would predict this strain to produce the poly(Rbo-P) WTA variant [8]. At the chromosomal location that harbors the WCFS1 taglocus, the JDM1 chromosome contains a stretch of 160 bp that is not present in the WCFS1 genome (Figure 2B).

\section{Genetic engineering results in the biosynthesis of alternative WTA variants}

Gene deletion mutants were exploited to assess the capability of $L$. plantarum WCFS1 to produce alternative WTA variants. To study the consequences of WTA removal, we used a recently described mutant in which the single copy of $\operatorname{tagO}$ was deleted [15]. Notably, the LTA backbone is synthesized via a completely independent pathway and, therefore, its biosynthesis is anticipated 


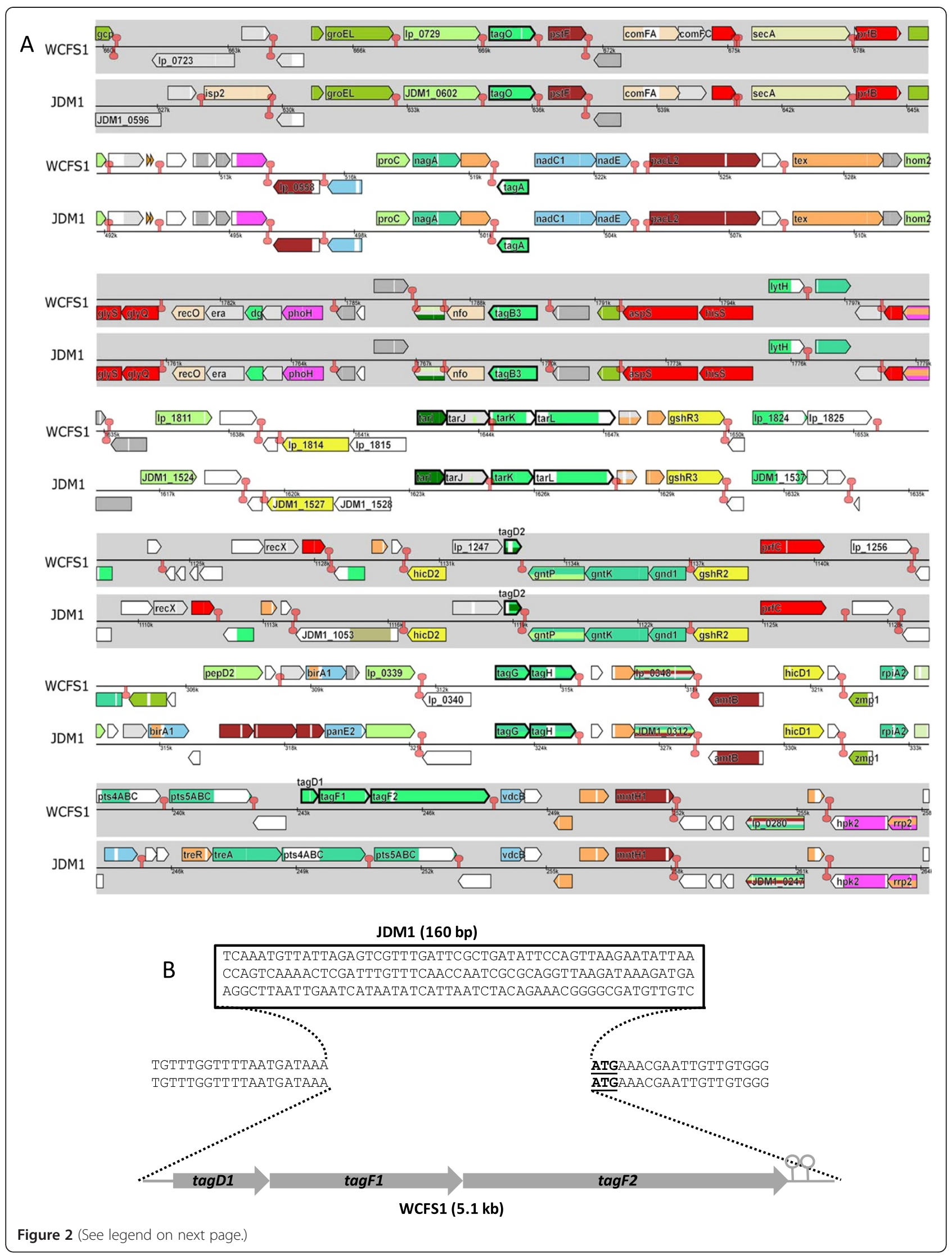


(See figure on previous page.)

Figure $\mathbf{2}$ Chromosomal organization of the 7 distinct genetic loci (represented by the iterating grey- and unshaded boxes) harboring the tag and tar genes in the L. plantarum WCFS1 and JDM-1 genomes (panel A). The tag and tar genes are highlighted in bold and named according to the current annotation in the WCFS1 genome. Colors represent different functional classes as defined by the microbial genome viewer tool [66]. Panel B displays detailed sequence information around the tagD1-tagF1-tagF2 locus (last grey-shaded box in Panel A) with the start codon of the downstream-encoded Ip_0271 (vdcB) underscored.

to be unaltered in $\operatorname{tagO}$ mutants [5]. In addition, the Gro-P polymerases (encoded by tagF) were targeted by mutagenesis, thereby anticipating to block the production of a poly(Gro-P)-containing WTA backbone (Figure 1), while maintaining the capability to synthesize a Gro-Pcontaining linkage unit (TagB activity) that is likely to be also involved in biosynthesis of a poly(Rbo-P) WTA $[5,9,37]$. Although two genes are annotated as $\operatorname{tag} F$, the size difference between the encoded TagF1 (lp_0268, 402 residues) and TagF2 (lp_0269, 941 residues) suggests that TagF1 might in fact encode an additional Gro-P primase (TagB) [9]. Nevertheless, as the function of TagF1 is currently unestablished, a tagF1-F2 mutant that lacks both genes was constructed. Lastly, a stable mutant was constructed in which the complete $\operatorname{dlt} X-D$ operon was deleted, aiming to produce WTA (and LTA [31]) that lacks D-Ala substitution. Notably, an L. plantarum mutant unable to substitute LTA with D-Ala residues has previously been described. However, this mutant harbored a $d l t B$ gene disruption obtained by single cross over plasmid integration within the $d l t B$ coding region, and as a consequence is genetically unstable [31]. Importantly, our dltX-D mutant displayed identical alterations in its basic physiological characteristics as the previously constructed $d l t B$ mutant $[31,38]$, including a lower maximum growth rate and final biomass, as well as cell lysis after prolonged stationary phase incubation (data not shown). Furthermore, the tagO mutant was already reported to display the tendency to remain associated in chains, and its cells appeared swollen, bent, and/or shorter than the wild-type [15]. By contrast, the growth and morphological characteristics of the newly constructed tagF1-2 mutant closely resembled the wildtype strain (data not shown).

To assess alditol composition, as well as glucosyl and D-Ala content, WTA was purified from the wild-type $L$. plantarum WCFS1 strain, and its tagO, tagF1-2, and dlt $X-D$ gene deletion derivatives (Figure 3 and Table 1). No WTA could be isolated from the $\operatorname{tag} O$ mutant, confirming earlier observations [15] that disruption of the first gene in the WTA biosynthetic pathway completely blocks WTA production in L. plantarum. From the other strains WTA isolation was successful and their phosphoric content was highly comparable, ranging from 2.0-2.3 $\mu \mathrm{mol} \mathrm{mg}{ }^{-1}$ (Table 1). The chemical composition analysis of WTA purified from the wild-type and the dlt $X-D$ mutant established the presence of a poly(Gro-P) backbone (Figure 3), as well as the presence of glucosyl

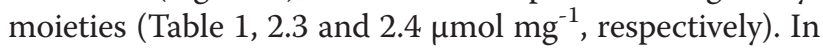
contrast to the wild-type $\left(0.79 \mu \mathrm{mol} \mathrm{mg} \mathrm{mg}^{-1}\right)$, no D-Ala could be detected in the purified WTA obtained from the $d l t X-D$ mutant, establishing the role of $\operatorname{dlt} X-D$ in D-alanylation of $L$. plantarum WTA next to its previously described role in D-Ala substitution of LTA [31]. The chemical analysis of WTA purified from the tagF1-2 mutant completely lacked the glycerol-specific signal, whereas (anhydro)ribitol could clearly be detected (Figure 3). These data demonstrate that the tagF1-2 mutant of L. plantarum WCFS1 is capable of producing WTA containing a poly(Rbo-P) backbone as an alternative for the poly(Gro-P) backbone produced by the parental strain. Notably, the glucosyl content $\left(1.1 \mu \mathrm{mol} \mathrm{mg}{ }^{-1}\right)$ and

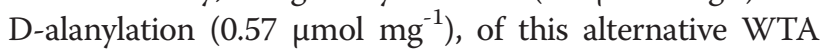
produced by the tagF1-2 mutant appeared to be reduced in comparison with the native poly(Gro-P) WTA produced by the wild-type. In addition, the relative abundance of poly(Rbo-P) WTA in the tagF1-2 mutant (32\% of total cell wall weight) was reduced compared to that of the poly (Gro-P) WTA in the wild-type (51\%) and dltX-D mutant (51\%) strains. Overall, the genetic engineering approach employed allowed the production of WTA variants in an isogenic L. plantarum background, and corroborates the inferences drawn from the in silico analysis.

To assess whether the production of alternative WTA molecules by the mutants impacts on either WTA or LTA polymer chain-length, purified WTA and LTA were utilized for high performance size exclusion chromatography (HPSEC) and proton NMR analyses, respectively (see the Additional file 1). These analyses reconfirmed that no WTA was produced by the $\operatorname{tagO}$ mutant, whereas the WTA produced by the $\operatorname{dlt} X-D$ mutant was of virtually identical backbone polymer length as compared to the WTA produced by the wild-type (Additional file 1: Table S1 in the supplementary information file). The WTA produced by the tagF1-2 mutant appeared to contain approximately $25 \%$ less backbone units (Rbo-P) as compared to the wild-type (Gro-P units). Similarly, LTA derived from the $\operatorname{tag} O$ and $d l t X-D$ mutants harbor a similar amount of Gro-P backbone units as compared to the wild-type, whereas the Gro-P LTA isolated from the tagF1-2 mutant appeared approximately 25\% lower (Additional file 1: Table S1 in the supplementary information file). Taken together, these data suggest that the described effects of WTA-pathway engineering have 

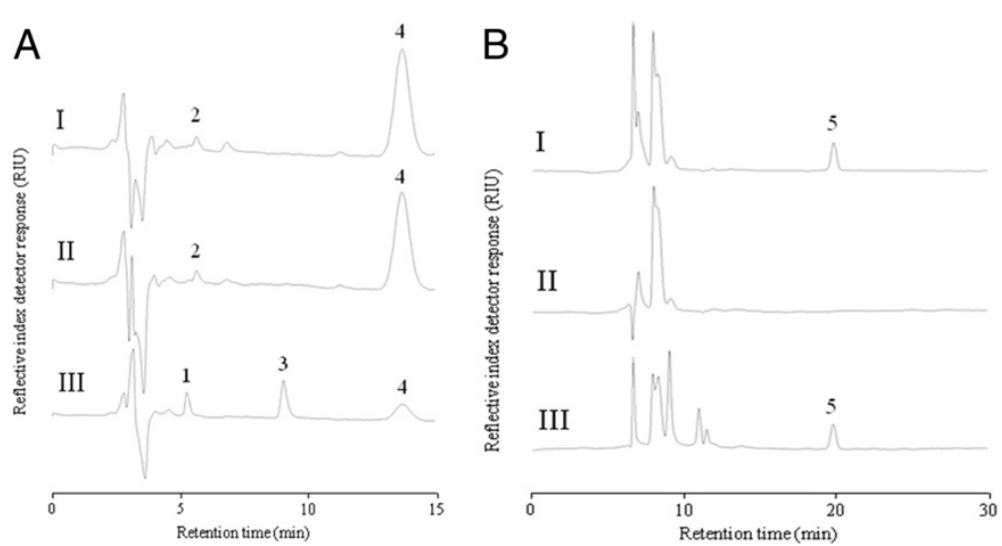

Figure 3 Sugars, alditols, and D-Ala composition of WTA isolated from L. plantarum WCFS1 (I), and the dItX-D (II), and tagF1-F2 (III) mutants. Panel A represents chromatograms on a Shodex NH2P-50 4E column, whereas panel B displays chromatograms on a Shodex SUGAR SC1011 column. Using pure compounds, peaks 1, 2, 3, 4 and 5 were identified to represent anhydroribitol, glycerol, ribitol, glucose, and alanine, respectively. The experiment is representative of 2 HPLC analyses.

relatively small effects on backbone chain length of WTA and LTA.

\section{Transcriptome analyses of mutants producing altered WTA}

To assess the impact of the production of the alternative WTA molecules on overall cell physiology, and specifically the WTA biosynthetic routes, whole-genome transcriptome profiles were generated for the dltX-D and tagF1-2 mutants and compared to the wild-type (Table 2 for genes involved in WTA biosynthesis and Additional file 2: Table S2 for all significantly regulated genes). As anticipated, no significant expression of the tagF1-2 and dltX-D genes could be detected in the corresponding mutants, verifying the expected mutation (Table 2). Remarkably, 230 and 402 genes appeared to display at least 2-fold altered transcription levels in the $d l t X-D$ and tagF1-2 mutants, including 30 and 47 genes that are annotated in the main functional class "cell envelope", respectively (Additional file 2: Table S2). These data strongly suggest compensatory behavior of $L$. plantarum upon modification of the cell wall component WTA (either lack of D-alanylation or alditol backbone switching), emphasizing the pivotal role WTA plays in the overall cell physiology of L. plantarum [15].

Interestingly, the $d l t X-D$ mutation led to a 3-fold induced expression of the tagGH-encoded WTA transporter
(Table 2). In addition, this mutant strain also expressed the tar-locus (tarIJKL) at a 7-fold higher level as compared to the wild-type, suggesting that the $\operatorname{dlt} X-D$ mutant may produce minute quantities of the poly(Rbo-P) WTA that remained undetected in the biochemical analyses, next to the dominant poly(Gro-P) WTA. When compared to the wild-type, the tarIJKL operon appeared among the strongest induced transcripts (up to 390-fold) identified in the transcriptome of the tagF1-2 mutant (Table 2). This finding in combination with the in silico and biochemical analyses described above establishes a definite role for tarIJKL in the production of the alternative poly(Rbo-P) WTA backbone in L. plantarum WCFS1. Notably, an ABCtransporter encoded by lp_0298-lp_0299 represents another genetic locus that displays strongly elevated expression levels in the tagF1-2 mutant as compared to the wild-type (Additional file 2: Table S2, 291- and 1049-fold, respectively). Although the substrate for this transporter is unknown, its elevated expression might indicate that it is involved in import of Rbo-P precursors or export of the poly(Rbo-P) WTA. In addition, several genes involved in production of the linkage unit $(\operatorname{tag} O, \operatorname{tag} A$, and tagD2) and the TA-transporter, predicted to be encoded by $\operatorname{tag} G H$, appeared induced in the poly(Rbo-P) WTA producing tagF1-2 mutant (Table 2). Despite the fact that these tag and tar genes displayed elevated

Table 1 Quantitative WTA analysis of L. plantarum WCFS1, and the tagF1-2 and dltX-D mutants

\begin{tabular}{|c|c|c|c|c|c|c|c|}
\hline \multirow[b]{2}{*}{ Strain: } & \multicolumn{7}{|c|}{ Phosphorus content $\left(\mu \mathrm{mol} \mathrm{mg}^{-1}\right)$ : } \\
\hline & WTA: & Cell wall: & $\%$ WTA (w/w): & D-Ala $\left(\mu \mathrm{mol} \mathrm{mg}{ }^{-1}\right)$ : & D-glucose $\left(\mu \mathrm{mol} \mathrm{mg}^{-1}\right)$ : & D-Ala / P: & D-glucose / P: \\
\hline wild-type & 2.0 & 1.0 & 51 & 0.79 & 2.3 & 0.39 & 1.1 \\
\hline$\Delta d l t X-D(\mathrm{NZ3539Cm})$ & 2.3 & 1.2 & 51 & N.D. & 2.4 & 0.0 & 1.0 \\
\hline$\overline{\Delta t a g F 1-2 ~(N Z 3547 C m)}$ & 2.2 & 0.72 & 32 & 0.57 & 1.1 & 0.25 & 0.51 \\
\hline
\end{tabular}

N.D. $=$ not detectable $\left(<0.01 \mu \mathrm{mol} \mathrm{mg}{ }^{-1}\right)$. The data is representative for 2 independent HPLC analyses. 
Table 2 Relative transcription levels of genes involved in WTA biosynthesis and D-Ala and glycosyl substitution in the dltX-D (NZ3539Cm) and tagF1-2 (NZ3547Cm) mutants as compared to the wild-type strain

\begin{tabular}{|c|c|c|c|c|c|}
\hline Ip number: & Gene name: & NZ3539Cm/WCFS1: & fdr: & NZ3547Cm/WCFS1: & fdr: \\
\hline Ip_0267 & tagD1 & 1.139 & 0.495 & 0.302 & 0.000 \\
\hline Ip_0268 & $\operatorname{tagF1}$ & 1.274 & 0.470 & 0.003 & 0.000 \\
\hline IP_0269 & $\operatorname{tag} F 2$ & 1.554 & 0.421 & 0.000 & 0.000 \\
\hline Ip_0343 & $\operatorname{tag} G$ & 2.667 & 0.000 & 2.394 & 0.001 \\
\hline IP_0344 & $\operatorname{tag} H$ & 3.386 & 0.000 & 2.380 & 0.001 \\
\hline IP_0564 & $\operatorname{tag} A$ & 1.814 & 0.013 & 1.501 & 0.120 \\
\hline Ip_0730 & $\operatorname{tag} O$ & 1.071 & 0.921 & 3.243 & 0.000 \\
\hline Ip_1248 & $\operatorname{tagD2}$ & 1.163 & 0.476 & 1.228 & 0.324 \\
\hline Ip_1816 & tarl & 6.638 & 0.006 & 317.9 & 0.000 \\
\hline IP_1817 & tars & 7.145 & 0.004 & 390.0 & 0.000 \\
\hline Ip_1818 & tark & 1.253 & 0.452 & 15.42 & 0.000 \\
\hline IP_1819 & $\operatorname{tarL}$ & 2.291 & 0.067 & 48.23 & 0.000 \\
\hline IP_1977 & $\operatorname{tag} B 3$ & 1.107 & 0.964 & 0.885 & 0.631 \\
\hline Ip_2016 & $d l t D$ & 0.001 & 0.000 & 0.792 & 0.245 \\
\hline Ip_2017 & dltCl & 0.002 & 0.000 & 0.545 & 0.031 \\
\hline Ip_2018 & $d \mid t B$ & 0.001 & 0.000 & 0.855 & 0.504 \\
\hline Ip_2019 & $d \mid t A$ & 0.068 & 0.000 & 0.286 & 0.006 \\
\hline IP_2020 & $d \mid t X$ & 0.207 & 0.000 & 0.905 & 0.706 \\
\hline $1 p_{-} 1406$ & $d \mid t C 2$ & 1.157 & 0.617 & 0.540 & 0.068 \\
\hline
\end{tabular}

transcription levels in the tagF1-2 mutant, the amount of WTA this strain produced is lower than that of the wildtype (see above), suggesting a lower overall efficacy of poly(Rbo-P) WTA biosynthesis and biogenesis as compared to its poly(Gro-P) counterpart. In addition, the dlt $X-D$ operon expression level appeared lower in the tagF1-2 mutant, which is in agreement with the reduced level of D-Ala substitution of the poly(Rbo-P) WTA relative to the poly(Gro-P) WTA produced by the wild-type. The fact that poly(Rbo-P) WTA biosynthesis utilizes alternative precursors was also clearly reflected in the transcriptome profiles. For example, the expression of the Gro-P-ABC transporter (lp_1324-1327), as well as tagD1 (lp_0267), involved in UDP activation of Gro-P, was decreased in the tagF1-2 mutant (Figure 4). Moreover, virtually all genes responsible for the import and conversion of both ribose and arabinose to CDP-Rbo displayed increased expression levels in the tagF1-2 mutant, whereas several of the surrounding pathways bridging to synthesis of other sugar-intermediates and glycolysis were downregulated (Figure 4).

The tagE genes encoded in the L. plantarum genome might be involved in glucosyl substitution of the poly (Rbo-P) WTA, based on their homology to TagE and TarM of B. subtilis and S. aureus, respectively $[39,40]$. This notion is supported by the approximately 1.8 -fold increased expression levels of tagE1, tagE3, and tagE6 homologues, in the tagF1-2 mutant. In addition, several other genes annotated as glycosyltransferases were either up- (lp_2845 and $l p \_3050 ; 17$ - and 5-fold, respectively) or down-regulated ( $l p_{-} 0304$ and $l p_{-} 1763$; both 4 -fold). This indicates that an additional or complementary role of these genes, but also glysosyl-transferases that displayed unaltered expression levels, in glycosyl substitution of either of the two WTA variants can certainly not be excluded.

\section{WTA backbone switching does not occur under laboratory conditions}

The experiments above unambiguously establish mutation of the tagF1-2 locus in L. plantarum WCFS1 can induce the biosynthesis of WTA containing the alternative poly(Rbo-P) backbone alditol, while the hyperinduction of the tarIJKL locus is a genetic marker for this event. Importantly, sequence analysis of the promoter upstream of the tarIJKL operon in the tagF1-2 mutant revealed the induced transcription of tarIJKL is not caused by promoter mutation, implying that the regulatory mechanism for WTA backbone alditol switching is intact in $L$. plantarum WCFS1. Therefore, it might be possible to induce WTA backbone alditol switching by modification of environmental conditions during growth. Hence, we attempted to identify fermentation conditions that lead to natural WTA alditol backbone switching. Firstly, we analyzed 29 transcriptome profiles generated previously in our laboratory in which varying temperature and $\mathrm{pH}$ were 


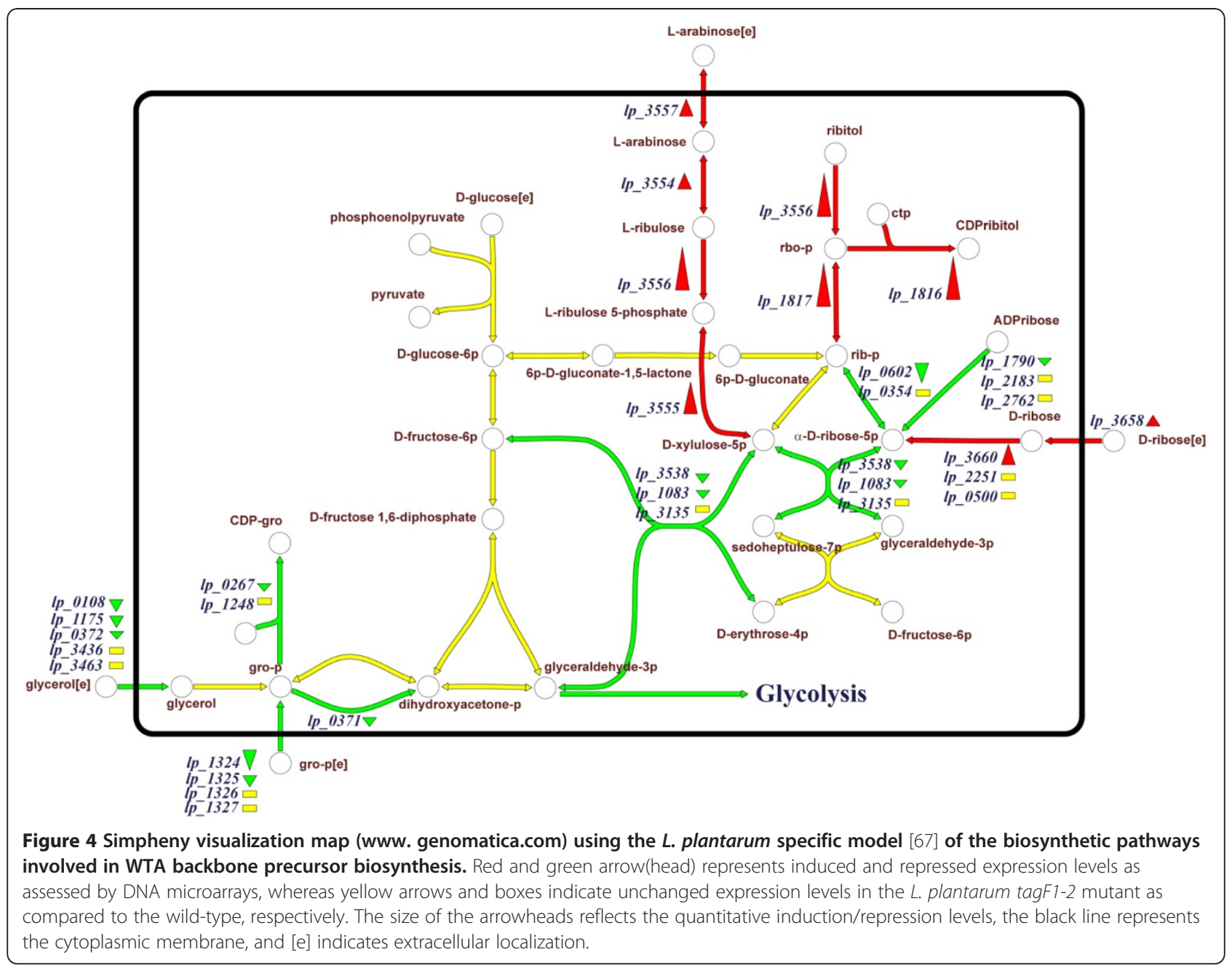

used, as well as different concentrations of amino acids, oxygen, and $\mathrm{NaCl}$ [41]. However, expression of the tarIJKL locus appeared extremely low under all these conditions (Additional file 3: Figure S1), suggesting that $L$. plantarum WCFS1 consistently produces the poly(Gro-P)-containing WTA backbone in these fermentations previously performed in our laboratory. Secondly, the observation that the transcriptome profiles generated for the tagF1-2 mutant (see above) revealed the induction of virtually all genes involved in the import and conversion of ribose and arabinose (Figure 4), triggered us to ferment $L$. plantarum WCFS1 using alternative sugars as carbon sources. Cells were harvested from logarithmic phase after growth on sucrose or ribose as sole carbon source, and RNA was isolated for qRT-PCR analyses, revealing low tarIJKL expression levels, highly comparable to $L$. plantarum grown on glucose which is established to produce a Gro-Pcontaining WTA by our biochemical analyses. Notably, bacterial growth on arabinose was insufficient to perform accurate qRT-PCR analysis of the tar locus. Therefore, the natural conditions resulting in WTA backbone alditol switching, if any, remain to be established. Taken together, these data indicate that the regulatory circuit required for the WTA alditol backbone switch is functional in L. plantarum WCFS1, but the natural conditions leading to alditol-switching remain to be identified. In the tagF1-2 mutant background 35 regulators were differentially expressed compared to the wild type strain (Additional file 2: Table S2), suggesting that one or more of these regulators may play a role in the regulation of alditol-switching of the WTA biosynthesis pathway.

\section{The role of WTA in immune signaling and modulation}

To identify the role of TLR-2/6 heterodimers in WTA immune signaling, the NF- $k B$ pathway activating capacity of purified WTAs and the L. plantarum TA mutants were assessed in a HEK-reporter cell line that expresses TLR-2/6. Exposure of this HEK-reporter cell line to wild-type L. plantarum cells activated TLR2/6 induced NF- $\mathrm{kB}$, whereas virtually no activation was measured with the $d l t X-D$ mutant (Figure $5 \mathrm{~A}$ ), which resembles earlier observations by Grangette et al... using a $L$. 


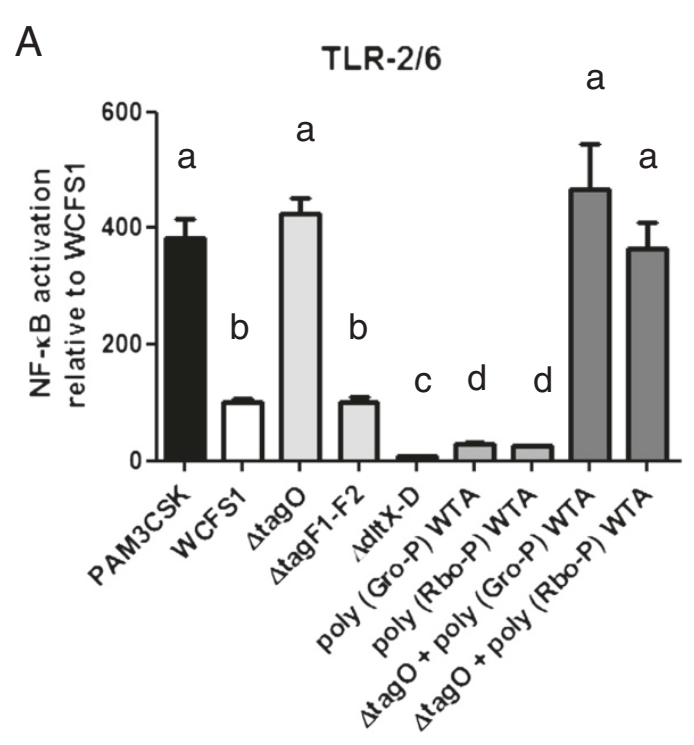

B

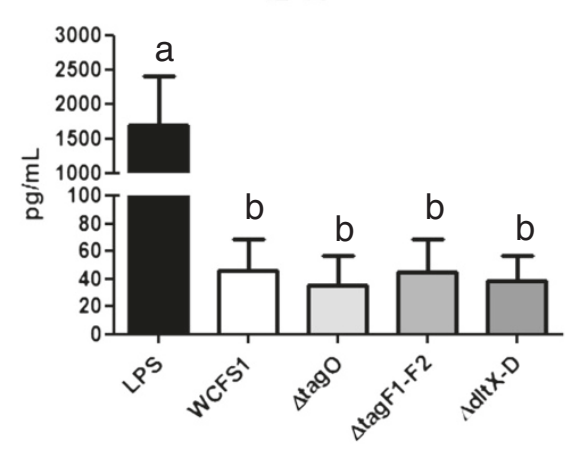

TNF- $\alpha$

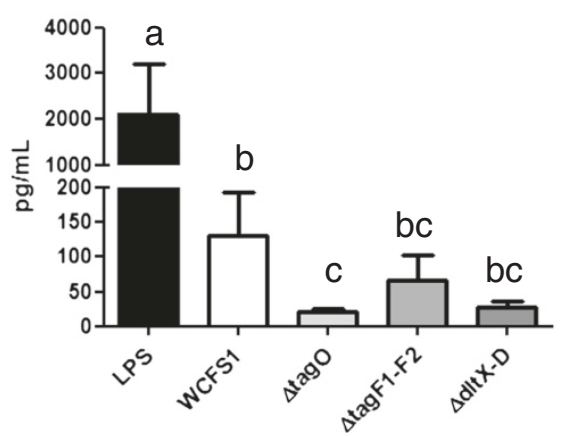

IL-12p70

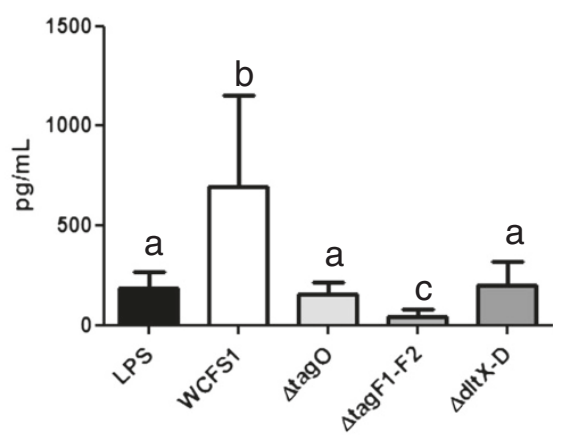

Figure $\mathbf{5}$ Immunomodulating and signaling capacity of the L. plantarum TA mutants. Panel A displays NF-KB pathway activation relative to L. plantarum WCFS1, as measured by a luminescence reporter in HEK cell lines expressing TLR-2/6 after exposure to PAM3CSK (positive control), purified WTA (5.0 $\mathrm{mg} / \mathrm{ml}$, see Additional file 4: Figure S2 and Additional file 5: Figure S3 for concentration ranges) and/or L. plantarum WCFS1, the tagO, tagF1-2, or dltX-D mutants. The ratios of HEK reporter to bacterial cell were 1:15. All strains were grown in triplicate and each culture was applied three times to the assay. Bars represent averages of these nine measurements with standard deviations. Letters a-d represent classes of statistically different responses as determined by ANOVA multiple comparison. Panel B displays cytokine levels secreted by monocyte derived iDCS stimulated with LPS (positive control), L. plantarum WCFS1, and the tagO, tagF1-2 and dltX-D mutants. The ratios of dendritic to bacterial cells were 1:10. All strains were grown in duplicate and each culture was applied in duplicate to the assay. Bars represent averages of these four measurements with standard deviations. Individual letters (a-c) represent classes of statistically significant different responses as determined by restricted maximum likelihood analysis, whereas "bc" represents a class not significantly different from "b" nor "c". 
plantarum dltB mutant and purified LTA [31], confirming the suitability of our signaling assay. These experiments indicate that TLR-2/6 signaling by intact $L$. plantarum WCFS1 cells depends on the presence of DAla substituted TAs on the cell surface. Furthermore, addition of a wide concentration range $(0.2-125 \mu \mathrm{g} / \mathrm{ml})$ of WTA purified from the wild-type [poly(Gro-P) backbone] and the tagF1-2 mutant [poly(Rbo-P) backbone] in the assays did not result in any signal above the medium control (Figure 5A for an exemplary WTA concentration and Additional file 4: Figure S2 for a large range of WTA concentrations), demonstrating that WTA has no direct role in TLR-2/6 signaling. Furthermore, TLR-2/6-mediated $\mathrm{NF}-\kappa \mathrm{B}$ activation by the tagF1-2 mutant was similar to the wild-type (Figure $5 \mathrm{~A}$ ), whereas the $\operatorname{tag} \mathrm{O}$ mutant displayed significantly increased signaling, suggesting that WTA deficiency might lead to increased exposure of TLR-2/6 agonists such as LTA, independent of the WTA backbone alditol. Attempts to functionally complement the $\operatorname{tag} O$ mutant by the addition of purified poly(Gro-P) or poly(Rbo-P) WTA, followed by exposure to the HEKreporter cell line, revealed enhanced signaling, highly similar to addition of the tagO mutant alone (Additional file 5: Figure S3, Figure 5), indicating that WTA is only capable of shielding TLR-2/6 signaling when it is localized appropriately in the cell wall.

To evaluate the immunomodulating characteristics of WTA, the cytokine production profiles of immature human monocyte derived dendritic cells (iDCs) were determined after stimulation with purified WTA and the mutant strains. Application of purified poly(Gro-P) or poly(Rbo-P) WTA to this assay resulted in undetectable interleukin (IL)-10, IL-12p70 and TNF- $\alpha$ secretion levels, comparable to iDCs to which medium was applied as a control $\left(<0.1 \mathrm{pg} \mathrm{ml}^{-1}\right.$, data not shown), suggesting that WTA is not directly involved in immunomodulation. The IL-10 levels secreted by DCs stimulated with all TA mutants were comparable to the levels obtained with the wild-type (Figure 5B). By contrast, levels of IL12 p70 were significantly lower for the $d l t X-D$ mutant as compared to the wild-type, suggesting that D-Ala substitution of TAs strongly contributes to the capacity of $L$. plantarum to elicit pro-inflammatory immune responses. The tagO mutant also elicited a decreased production of IL-12p70 as compared to the wild-type, indicating that, in addition to LTA [31], the presence of WTA impacts on IL-12p70 production levels. Moreover, the IL-12 production levels obtained upon exposure of immature DCs (iDCs) to the tagF1-2 mutant were even lower than those of the $\operatorname{dlt} X-D$ and $\operatorname{tag} O$ mutants, indicating that the biosynthesis of the alternative poly(Rbo-P)-containing WTA backbone has differential consequences for the immune response elicited by DCs, resulting in altered pro-inflammatory cytokine production. The observed reduction of IL-12 production levels in these DC assays was reflected (although significant only for $\operatorname{tag} O$ mutant) in the levels of TNF- $\alpha$ production in these assays.

\section{Discussion}

Several L. plantarum strains encode the genetic determinants for the production of WTA variants containing either poly(Gro-P) or poly(Rbo-P) backbones [8], whereas other lactobacilli appear to exclusively produce the poly (Gro-P) variant or produce no WTA at all $[2,3]$. LTA polymers consistently contain a glycerol backbone [29] that is synthesized via an independent pathway involving LtaS (LTA synthase)-dependent polymerization of phosphatidylglycerol, rather than the nucleotide-activated sugars utilized as precursors for WTA biosynthesis [5]. Moreover, LTA phosphatidylglycerol polymerization occurs directly onto a glycolipid that serves as the membrane anchor, rather than the undecaprenyl phosphate anchor employed in WTA biosynthesis $[5,18]$. Indeed, we were able to show here that modification of WTA had no impact on the type of LTA backbone produced (GroP), and only slightly altered the chain length in the case of the tagF1-2 mutant. The independence of these biosynthetic pathways allowed investigation of WTA-specific alditol switching in an isogenic background in L. plantarum WCFS1, as well as the assessment of the overall effects thereof on bacterial physiology and the WTA biosynthetic routes using a transcriptomics approach. In view of the substantial transcriptome responses elicited by the mutations introduced, it may be that TA engineering has some general effects for cell wall architecture and turn-over that go beyond the specific components that were targeted by mutagenesis here. This may elicit some effects in terms of host cell recognition and/or response, but the data presented clearly establish that immunomodulatory effects observed in this study are not directly influenced by the WTAs.

The work presented here functionally demonstrates a role for the tar-locus (tarIJKL) in the production of poly(Rbo-P) WTA in L. plantarum. Moreover, the DNA microarray experiments performed with $L$. plantarum WCFS1 and its derivatives producing alternative WTA variants indicate that ribose and/or arabinose may serve as the preferred carbohydrate sources for the production of the precursors for this alternative WTA backbone. However, we have not been able to identify fermentation conditions that lead to the natural switching of the WTA backbone. Reports on other Gram-positive bacteria such as $B$. subtilis and $S$. aureus describe the production of strain-specific WTA backbone alditols [5], as well as an $S$. aureus strain producing both poly(Gro-P) and poly (Rbo-P) WTA types simultaneously [42]. However, to the best of our knowledge, this is the first report presenting the genetic and functional capability of WTA alditolbackbone switching. Moreover, it also is the first study 
that analyzes the biosynthetic WTA pathway(s) in a probiotic species and evaluates the consequences for modulation of host immune cells and innate, TLR-2/6-mediated signaling.

A comparative in silico analysis of the poly(Gro-P) and poly(Rbo-P) associated WTA genes in the B. subtilis strains W23 and 168, respectively, suggests that at least some of the genes encoding poly(Gro-P) synthesis in strain 168 were acquired by horizontal gene transfer, possibly displacing all, or part of, the resident W23-like poly(Rbo-P) WTA biosynthesis genes [9]. Our in silico analysis suggested that a similar horizontal gene transfer event may have occurred in L. plantarum, whereby the genes involved in poly(Gro-P) WTA biosynthesis were acquired in an ancestral, poly(Rbo-P) WTA producing strain, while leaving the capacity to produce this native WTA variant intact. However, phylogenetic analysis of 18 L. plantarum strains revealed that the tarIJKL locus cluster in two groups correlating completely with the WTA backbone-type produced [8]. As it seems unlikely that the genes involved in poly(Rbo-P) WTA biosynthesis have co-evolved in strains producing poly(Gro-P) WTA, the exact order of evolutionary events remains unknown. Nevertheless, these events have led to a subset of L. plantarum strains that have the genetic potential to alternate their WTA biochemistry, which supports earlier suggestions that this canonical compound might play an important role in the lifestyle of this bacterium and may have a profound impact on its molecular communication with mucosal cells in the intestinal tract, i.e. its probiotic efficacy [2]. The observation that more poly(Gro-P) WTA is produced than poly(Rbo-P) WTA might present another possible explanation for the alditol switching, i.e. to produce higher quantities of this cell wall polymer under certain (yet unknown) stress conditions, possibly including adaptation to the host intestinal environment. To this end, it might also be interesting to investigate WTA alditol backbone switching by introducing the tag locus into strains producing Rbo-P type WTA.

The ability of peptidoglycan and other non-acylated cell wall components to induce TLR-2 signaling is controversial $[43,44]$, and some studies have highlighted contamination with lipoproteins as a confounding factor [45]. Notably, these studies typically investigated immunomodulation by analyzing purified components of the cell envelope, ignoring shielding effects [46] as well as the interplay between different components, which is highly likely to blur our view of the full immunomodulatory capacity of intact bacterial cells possessing their native extracellular architecture and composition. Therefore, we assessed the differential immune responses triggered by exposure to L. plantarum TA mutants and/or purified WTA, thereby conclusively showing that purified L. plantarum WTA of either backbone alditol type is unable to directly signal via TLR-2/6. Nevertheless, the WTA negative derivative displayed enhanced TLR-2/6 signaling, suggesting that WTA shields or prevents release of other immunomodulating compounds, such as LTA, a known agonist of TLR $2 / 6$, and thereby indirectly contributes to attenuation of TLR-2/6 signaling. In addition, our observation that deletion of the genes required for $\mathrm{D}$ alanylation of TA specifically reduces TLR-2/6 signaling, strongly suggests the TLR-2/6 heterodimer is involved in L. plantarum LTA signaling, in line with earlier experiments performed with Gram-positive pathogens $[47,48]$. Hence, our data fine-tune earlier murine-based observations revealing that L. plantarum LTA signaling is TLR-2dependent [31], to the exact human TLR heterodimer involved. Notably, the dltX-D mutant reported here produced LTA of a backbone chain length highly comparable to L. plantarum WCFS1, whereas the previously constructed $d l t B$ mutant mainly produced LTA that was approximately 3 times longer than that produced by the wild-type [38]. Although the reason for this apparent discrepancy is currently unknown, it may be explained by the different nature of the genetic mutation introduced in these strains. While the mutant presented in this study lacks the entire gene cluster encoding the Dlt machinery, the mutant described by Palumbo et al... carries a mutation only in the $d l t B$ gene. As a consequence, the remaining Dlt functions are likely still expressed in the latter mutant, which may influence the chain length determination of LTA, possibly by binding of Dlt components to the emerging LTA polymer. On the other hand, our results using the dlt $X-D$ mutant do corroborate a recent report that deletion of a phosphoglycerol transferase gene (ltaS), that plays a key role in LTA biosynthesis, in Lactobacillus acidophilus NCFM down-regulated IL-12 and TNF- $\alpha$ production in DCs in a TLR-2-dependent manner [30]. Conversely, the fact that the enhanced TLR-2/6 signaling of the tagO mutant observed in the HEK-reporter cell line that only expresses TLR-2/6 did not translate to elevated IL-12 and TNF- $\alpha$ levels in DCs equipped with the full arsenal of receptors, but in fact resulted in decreased levels of these cytokines, strengthens the concept that communication between probiotic lactobacilli and intestinal host cells is multi-factorial and involves an integrative repertoire of receptors on the host side that recognize multiple effector molecules on the bacterial side [49]. It remains to be established whether WTA is directly involved in signaling via other host receptors or, alternatively, shielding of immunomodulating compounds that signal via other receptors.

\section{Conclusions}

The data presented here has several consequences for research addressing molecular host-probiotic communication. The observation that the pro-inflammatory 
capacity (IL-12 levels) of L. plantarum WCFS1 depends on the amount and alditol backbone variant of WTA synthesized, implies that the extrapolation of data obtained from in vitro immunological assays to the in situ situation in the gastrointestinal tract is certainly not trivial. To this end, the relative level of production of WTA may be altered in situ, as has been shown for $S$. aureus when it resides in the nasal cavity of rats [50], but also here by the observation that WTA backbone switching results in differential levels of WTA produced (Table 1). Moreover, not all Lactobacillus species have the capacity to produce WTA, or may alter either WTA backbone type or WTA production levels under certain conditions [2,3], which could severely impact on their signaling capacity as compared to strains that do produce this molecule. The biochemical characteristics of TAs, such as polymer length and its exact composition, including the degree of D-Ala and glycosyl substitution, may fine-tune the immune signaling effects described here. In this respect, it is intriguing that Marco and coworkers have reported the significant down-regulation of the $d l t X-D$ operon during passage of $L$. plantarum through the murine gastrointestinal tract [51]. Overall, the flexibility of bacteria to produce different (ratios of) TAs that have alternative biochemical characteristics (backbone and/or substitutions) under different environmental conditions is bound to impact on their hostcommunication capacities in vivo [50,51]. Moreover, inter-strain differences in the production of specific TA molecules provide a plausible explanation for the remarkable strain-specificity observed in immune assays $[52,53]$. Taken together, this study correlates structural differences in TAs to their functional characteristics and provides important information to improve our understanding of molecular host-microbe interactions and probiotic functionality.

\section{Methods}

\section{Strains and culture conditions}

L. plantarum WCFS1 [19] and its derivatives were cultivated in Mann-Rogusa Sharpe (MRS) broth (Merck, Darmstadt, Germany) or $2 \times$ chemically defined medium containing $1.5 \%$ glucose [54] at $37^{\circ} \mathrm{C}$ without agitation. When appropriate, $10 \mu \mathrm{g} \mathrm{ml}^{-1}$ chloramphenicol or erythromycin was added. Escherichia coli MC1061 [55] was used as an intermediate cloning host for the construction of the mutagenesis vectors (see below). E. coli was grown aerobically at $37^{\circ} \mathrm{C}$ in TY medium, supplemented with $10 \mu \mathrm{g} \mathrm{ml}{ }^{-1}$ chloramphenicol when appropriate.

\section{Gene deletion mutant construction and DNA microarray analysis}

L. plantarum gene deletion mutant construction by a double cross-over gene replacement strategy for tagF1-F2 (lp_0268 \& lp_0269) and dltX-D (lp_2016-lp_2020) was performed essentially as described previously [56] using the primers presented in Additional file 1: Table S3 (included in the Additional file 1: supplementary materials and methods file). RNA isolation from L. plantarum, subsequent cDNA synthesis and indirect labeling, as well as DNA microarray hybridizations were performed using routine procedures [51,52]. Detailed experimental procedures are reported in the Additional file 1 . The custom-made 60-mer oligonucleotide array design (Agilent Biotechnologies, Amstelveen, The Netherlands) and whole-genome transcriptomics data of the WTA mutants are accessible in GEO as platform GPL9359 and dataset GSE27683.

\section{WTA preparation and analysis}

WTAs were prepared from cell walls of the L. plantarum strains as described previously [8]. Briefly, the cells were grown to the stationary growth phase in $8 \mathrm{~L}$ MRS for $20 \mathrm{~h}$ and harvested by centrifugation $(20,000 \times \mathrm{g}$ for $20 \mathrm{~min}$ at $4^{\circ} \mathrm{C}$ ). The cells were disrupted by ultrasonication and treated with $2 \%$ sodium dodecyl sulfate, Pronase (Roche Diagnostics, Mannheim, Germany), DNase (Wako Pure Chemical Industries, Osaka, Japan), RNase (Sigma-Aldrich, St. Louis, USA), and Pepsin (SigmaAldrich). After thorough washing with distilled water, cell-walls were pelleted by centrifugation $(20,000 \times \mathrm{g}$ for $20 \mathrm{~min}$ at $4^{\circ} \mathrm{C}$ ) and lyophilized. The WTAs were released from the cell walls by $10 \%$ trichloroacetic acid treatment at $4^{\circ} \mathrm{C}$, collected by ethanol precipitation at $4^{\circ} \mathrm{C}$, and hydrolyzed with $2 \mathrm{~N}$ hydrochloric acid at $100^{\circ} \mathrm{C}$ for $3 \mathrm{~h}$. Sugars and alditols in the WTA hydrolysates were identified using a high-performance liquid chromatography (HPLC, LC-10 system, Shimadzu, Kyoto, Japan) on a Shodex Asahipak NH2P-50 4E column (Showa Denko, Tokyo, Japan) with water-acetonitrile $(25: 75, \mathrm{v} / \mathrm{v})$ as the eluent at $30^{\circ} \mathrm{C}[8,57]$. The D-Ala content was quantified using the HPLC system on a Shodex SUGAR SC1011 column (Showa Denko) with $10 \mathrm{mM}$ calcium chloride ( $\mathrm{pH} \mathrm{5.8)}$ as the eluent at $80^{\circ} \mathrm{C}$. Phosphoric acid, glucose, and D-Ala content in the WTAs were determined by Allen's method [58], phenol-sulfuric acid method [59], and HPLC, respectively.

\section{Innate signaling and immunological assays}

Buffy coats from 4 blood donors were obtained from the Sanquin blood bank (Nijmegen, The Netherlands). A written informed consent was obtained from each volunteer prior to sample collection. Monocyte isolation from human blood, generation of immature dendritic cells (iDCs), and stimulation of iDCs with LPS (final concentration of $1.0 \mu \mathrm{g} / \mathrm{ml}$, positive control) or L. plantarum strains (1:10 ratio, all strains in duplicate from 2 
independent cultures) was performed as described previously [52]. After 48h, duplicate supernatant from the DC stimulation assays were analyzed for the presence of cytokines (TNF- $\alpha$, IL-12p70 and IL-10) using a cytometric bead-based immunoassay that enables multiplex measurements of soluble cytokines in the same sample [60], according to the manufacturer's protocol (BD biosciences, Breda, The Netherlands). The limits of sensitivity for detection were: TNF- $\alpha 0.7 \mathrm{pg} \mathrm{m}^{-1}$; IL-12p70 $0.6 \mathrm{pg}$ $\mathrm{ml}^{-1}$ and IL-10 $0.13 \mathrm{pg} \mathrm{ml}^{-1}$. The flow cytometry data were analyzed using the BD FCAP software (BD biosciences, Breda, The Netherlands). For statistical analysis mixed general linear model using restricted maximum likelihood (REML) was used to determine the statistical differences within donors between cytokine produced by DCs stimulated with the constructed deletion mutants compared to the parental L. plantarum WCFS1 strain. A two-sided $p$-value of 0.05 or lower was considered to be significant. The statistical analysis was performed by using SAS software (version 9.1, SAS Institute Inc., Cary, NC, USA).

Human embryonic kidney (HEK)293 cells harboring pNIFTY, a NF- $\mathrm{kB}$ luciferase reporter construct (Invivogen, Toulouse, France) were used as the negative control in the NF- $\mathrm{kB}$ assays. A HEK293 cell line constitutively expressing human hTLR-2/6 was seeded at $5 \times 10^{5}$ cells $\mathrm{cm}^{-2}$ in 96 -well plates and incubated overnight under standard culture conditions [61]. Subsequently, these cell lines were incubated in triplicate for $6 \mathrm{~h}$ with 3 independent bacterial batches of L. plantarum WCFS1 or its derivatives (15 CFU/HEK293 cell), the positive control TLR2 ligand Pam3-Cys-SK4 (5 $\mu \mathrm{g} \mathrm{ml} \mathrm{ml}^{-1}$ ), or with medium alone as a negative control. NF- $\mathrm{kB}$ activation was measured by the Bright-glo luciferase assay (Promega Benelux BV, Leiden, The Netherlands). As anticipated, NF-kB activity significantly increased only in the cell lines transfected with hTLR-2/6. Values obtained with the L. plantarum strains were normalized to medium induced NF- $\mathrm{KB}$ activity. ANOVA multiple comparison was used to determine the statistical differences between the NF- $\kappa B$ induced activities stimulated with the constructed deletion mutants compared to the parental L. plantarum WCFS1 strain. A two-sided $p$-value $<0.05$ was considered significant.

The study was approved by the Wageningen University Ethical Committee and was performed according to the principles of the Declaration of Helsinki. Buffy coats from blood donors were obtained from the Sanquin Blood bank in Nijmegen (The Netherlands). A written informed consent was obtained from all blood donors prior to sample collection.

\section{Additional files} Additional file 1: Detailed description of mutant construction, DNA
microarray analysis, and LTA and WTA chain length determination.

Additional file 2: Table S2. Relative transcription levels of genes over 2-fold regulated (fdr < 0.05) in the ditX-D (NZ3539Cm) and tagF1-2 (NZ3547Cm) mutants as compared to the wild-type strain.

Additional file 3: Figure S1. Expression level of the tarlJKL operon (Ip_1816-1819) compared to the median of expression under a variety of fermentation conditions.

Additional file 4: Figure S2. The signaling capacity of purified WTAs was measured as NF-KB pathway activation relative to a medium only control, as measured by a luminescence reporter in HEK cell lines expressing TLR-2/6 after exposure to $0.2,1.0,5.0,25$ or $125 \mu \mathrm{g} / \mathrm{ml} \mathrm{WTA}$ containing either a poly(Gro-P) or poly(Rbo-P) backbone (isolated from $L$. plantarum WCFS1 or the tagF1-2 mutant, respectively). All WTAs were applied three times to the assay. Bars represent averages of these three measurements with standard deviations.

Additional file 5: Figure S3. The signaling capacity of the tagO mutant functionally complemented with WTA was measured as NF-kB pathway activation relative to the tagO mutant without added WTA, as measured by a luminescence reporter in HEK cell lines expressing TLR-2/6. HEK cell lines were exposed to the tagO mutant (The ratios of HEK reporter to bacterial cell were 1:15) plus $0.2,1.0,5.0,25$ or $125 \mu \mathrm{g} / \mathrm{ml}$ WTA containing either a poly(Gro-P) or poly(Rbo-P) backbone (isolated from L. plantarum WCFS1 or the tagF1-2 mutant, respectively). All samples were applied three times to the assay, and the bars represent averages of these three measurements with standard deviations.

\section{Competing interests}

The authors declare that they have no competing interests.

\section{Authors' contributions}

PAB concepted the study, and drafted the manuscript. ST performed the WTA and LTA analyses and critically revised the manuscript. IIVS constructed the tagF1-2 mutant and performed the qRT-PCR experiments. DMR performed the DNA microarray experiments and critically revised the manuscript. MM designed and performed the signalling and immune assays, and critically revised the manuscript. MW designed the DNA microarray experiments, and performed the data analysis and visualization. SO designed the WTA and LTA analyses. JMW designed the immune and signalling experiments, and critically revised the manuscript. MK conceived the study and drafted the manuscript. All authors read and approved the final manuscript.

\section{Acknowledgements}

We cordially thank Nico Taverne and Roger Bongers for technical assistance with the immunological assays and the DNA microarray experiments, respectively. We acknowledge the role of our project leader Dr. Paul de Vos (University Medical Centre Groningen, The Netherlands) and Dr. Eiichi Satoh (Tokyo University of Agriculture, Japan) in coordinating and facilitating the work performed.

\section{Author details}

${ }^{1}$ TI Food \& Nutrition, Nieuwe Kanaal 9A, 6709PA, Wageningen, The Netherlands. ${ }^{2}$ NIZO Food Research, Kernhemseweg 2, 6718ZB, Ede, The Netherlands. ${ }^{3}$ Kluyver Centre for Genomics of Industrial Fermentation, Julianalaan 67, 2628 BC, Delft, The Netherlands. ${ }^{4}$ Department of Applied Biology and Chemistry, Faculty of Applied Bio-Science, Tokyo University of Agriculture, 1-1-1 Sakuragaoka, Setagaya-ku, Tokyo 156-8502, Japan. ${ }^{5}$ Host-Microbe Interactomics, Wageningen University, Marijkeweg 40, 6709 PG, Wageningen, The Netherlands. ${ }^{6}$ Netherlands Consortium for Systems Biology, Science Park 904, 1098 XH, Amsterdam, The Netherlands.

${ }^{7}$ Laboratory for Microbiology, Wageningen University, Dreijenplein 10, 6703 $H B$, Wageningen, The Netherlands.

Received: 2 March 2012 Accepted: 28 July 2012 Published: 11 September 2012 


\section{References}

1. Vollmer W: Structural variation in the glycan strands of bacterial peptidoglycan. FEMS Microbiol Rev 2008, 32(2):287-306.

2. Kleerebezem M, Hols P, Bernard E, Rolain T, Zhou M, Siezen RJ, Bron PA: The extracellular biology of the lactobacilli. FEMS Microbiol Rev 2010, 34(2):199-230

3. Bron $\mathrm{PA}$, van Baarlen $\mathrm{P}$, Kleerebezem $\mathrm{M}$ : Emerging molecular insights into the interaction between probiotics and the host intestinal mucosa. Nat Rev Microbiol 2012, 10:66-78.

4. Lebeer S, Vanderleyden J, De Keersmaecker SC: Host interactions of probiotic bacterial surface molecules: comparison with commensals and pathogens. Nat Rev Microbiol 2010, 8(3):171-184.

5. Xia G, Kohler T, Peschel A: The wall teichoic acid and lipoteichoic acid polymers of Staphylococcus aureus. Int I Med Microbiol 2010, 300(2-3):148-154.

6. Fischer W: Physiology of lipoteichoic acids in bacteria. Adv Microb Physiol 1988, 29:233-302.

7. Jang KS, Baik JE, Han SH, Chung DK, Kim BG: Multi-spectrometric analyses of lipoteichoic acids isolated from Lactobacillus plantarum. Biochem Biophys Res Commun 2011, 407(4):823-830.

8. Tomita S, Irisawa T, Tanaka N, Nukada T, Satoh E, Uchimura T, Okada S: Comparison of components and synthesis genes of cell wall teichoic acid among Lactobacillus plantarum strains. Biosci Biotechnol Biochem 2010, 74(5):928-933.

9. Lazarevic V, Abellan FX, Moller SB, Karamata D, Mauel C: Comparison of ribitol and glycerol teichoic acid genes in Bacillus subtilis W23 and 168: identical function, similar divergent organization, but different regulation. Microbiology 2002, 148(Pt 3):815-824.

10. Brown $\mathrm{S}$, Zhang $\mathrm{YH}$, Walker S: A revised pathway proposed for Staphylococcus aureus wall teichoic acid biosynthesis based on in vitro reconstitution of the intracellular steps. Chem Biol 2008, 15(1):12-21.

11. Bhavsar AP, Beveridge TJ, Brown ED: Precise deletion of tagD and controlled depletion of its product, glycerol 3-phosphate cytidylyltransferase, leads to irregular morphology and lysis of Bacillus subtilis grown at physiological temperature. J Bacteriol 2001, 183(22):6688-6693.

12. Bhavsar AP, Erdman LK, Schertzer JW, Brown ED: Teichoic acid is an essential polymer in Bacillus subtilis that is functionally distinct from teichuronic acid. J Bacteriol 2004, 186(23):7865-7873.

13. D'Elia MA, Pereira MP, Chung YS, Zhao W, Chau A, Kenney TJ, Sulavik MC, Black TA, Brown ED: Lesions in teichoic acid biosynthesis in Staphylococcus aureus lead to a lethal gain of function in the otherwise dispensable pathway. J Bacteriol 2006, 188(12):4183-4189.

14. Weidenmaier C, Kokai-Kun JF, Kristian SA, Chanturiya T, Kalbacher H, Gross M, Nicholson G, Neumeister B, Mond JJ, Peschel A: Role of teichoic acids in Staphylococcus aureus nasal colonization, a major risk factor in nosocomial infections. Nat Med 2004, 10(3):243-245

15. Andre G, Deghorain M, Bron PA, van Swam II, Kleerebezem M, Hols P, Dufrene YF: Fluorescence and atomic force microscopy imaging of wall teichoic acids in Lactobacillus plantarum. ACS Chem Biol 2011, 6(4):366-376.

16. D'Elia MA, Millar KE, Beveridge TJ, Brown ED: Wall teichoic acid polymers are dispensable for cell viability in Bacillus subtilis. J Bacteriol 2006, 188(23):8313-8316.

17. Delcour J, Ferain T, Deghorain M, Palumbo E, Hols P: The biosynthesis and functionality of the cell-wall of lactic acid bacteria. Antonie Van Leeuwenhoek 1999, 76(1-4):159-184.

18. Schirner K, Marles-Wright J, Lewis RJ, Errington J: Distinct and essential morphogenic functions for wall- and lipo-teichoic acids in Bacillus subtilis. EMBO J 2009, 28(7):830-842.

19. Kleerebezem M, Boekhorst J, van Kranenburg R, Molenaar D, Kuipers OP, Leer R, Tarchini R, Peters SA, Sandbrink HM, Fiers MW, et al: Complete genome sequence of Lactobacillus plantarum WCFS1. Proc Natl Acad Sci U S A 2003, 100(4):1990-1995

20. Vesa T, Pochart P, Marteau P: Pharmacokinetics of Lactobacillus plantarum NCIMB 8826, Lactobacillus fermentum KLD, and Lactococcus lactis MG 1363 in the human gastrointestinal tract. Aliment Pharmacol Ther 2000, 14(6):823-828

21. Adlerberth I, Ahrne S, Johansson ML, Molin G, Hanson LA, Wold AE: A mannose-specific adherence mechanism in Lactobacillus plantarum conferring binding to the human colonic cell line HT-29. Appl Environ Microbiol 1996, 62(7):2244-2251.

22. de Vries MC, Vaughan EE, Kleerebezem M, de Vos WM: Lactobacillus plantarum-survival, functional and potential probiotic properties in the human intestinal tract. Int Dairy J 2005, 16:1018-1028.

23. van Baarlen P. Troost FJ, van Hemert S, van der Meer C, de Vos WM, de Groot PJ, Hooiveld GJ, Brummer RJ, Kleerebezem M: Differential NF-kappaB pathways induction by Lactobacillus plantarum in the duodenum of healthy humans correlating with immune tolerance. Proc Natl Acad Sci U S A 2009, 106(7):2371-2376.

24. Macho Fernandez E, Pot B, Grangette C: Beneficial effect of probiotics in IBD: Are peptidogycan and NOD2 the molecular key effectors? Gut Microbes 2011, 2:5

25. Macho Fernandez E, Valenti V, Rockel C, Hermann C, Pot B, Boneca IG, Grangette C: Anti-inflammatory capacity of selected lactobacilli in experimental colitis is driven by NOD2-mediated recognition of a specific peptidoglycan-derived muropeptide. Gut 2011 60(8):1050-1059

26. Wells JM, Rossi O, Meijerink M, van Baarlen P: Epithelial crosstalk at the microbiota-mucosal interface. Proc Natl Acad Sci U S A 2011 , 108(Suppl 1):4607-4614.

27. Jin MS, Kim SE, Heo JY, Lee ME, Kim HM, Paik SG, Lee H, Lee JO: Crystal structure of the TLR1-TLR2 heterodimer induced by binding of a tri-acylated lipopeptide. Cell 2007, 130(6):1071-1082.

28. Claes IJ, Lebeer S, Shen C, Verhoeven TL, Dilissen E, De Hertogh G, Bullens DM, Ceuppens JL, Van Assche G, Vermeire S, et al: Impact of lipoteichoic acid modification on the performance of the probiotic Lactobacillus rhamnosus GG in experimental colitis. Clin Exp Immunol 2010, 60:1050-1059

29. Lebeer S, Claes IJ, Vanderleyden J: Anti-inflammatory potential of probiotics: lipoteichoic acid makes a difference. Trends Microbiol 2012, 20(1):5-10.

30. Mohamadzadeh M, Pfeiler EA, Brown JB, Zadeh M, Gramarossa M, Managlia E, Bere P, Sarraj B, Khan MW, Pakanati KC, et al: Microbes and Health Sackler Colloquium: Regulation of induced colonic inflammation by Lactobacillus acidophilus deficient in lipoteichoic acid. Proc Natl Acad SC U S A 2011, 15(108):4623-30.

31. Grangette C, Nutten S, Palumbo E, Morath S, Hermann C, Dewulf J, Pot B, Hartung T, Hols P, Mercenier A: Enhanced antiinflammatory capacity of a Lactobacillus plantarum mutant synthesizing modified teichoic acids. Proc Natl Acad Sci U S A 2005, 102(29):10321-10326.

32. Rockel C, Hartung T, Hermann C: Different Staphylococcus aureus whole bacteria mutated in putative pro-inflammatory membrane components have similar cytokine inducing activity. Immunobiology 2011, 216(3):316-321.

33. Kaji R, Kiyoshima-Shibata J, Nagaoka M, Nanno M, Shida K: Bacterial teichoic acids reverse predominant IL-12 production induced by certain lactobacillus strains into predominant IL-10 production via TLR2dependent ERK activation in macrophages. J Immunol 2010 184(7):3505-3513.

34. Siezen RJ, Francke C, Renckens B, Boekhorst J, Wels M, Kleerebezem M, van Hijum SA: Complete Resequencing and Reannotation of the Lactobacillus plantarum WCFS1 Genome. J Bacteriol 2012, 194(1):195-196.

35. Zhang ZY, Liu C, Zhu YZ, Zhong Y, Zhu YQ, Zheng HJ, Zhao GP, Wang SY, Guo XK: Complete genome sequence of Lactobacillus plantarum JDM1. J Bacteriol 2009, 191(15):5020-5021.

36. Zhang Z, Schwartz S, Wagner L, Miller W: A greedy algorithm for aligning DNA sequences. J Comput Biol 2000, 7(1-2):203-214.

37. Kojima N, Araki Y, Ito E: Structural studies on the linkage unit of ribitol teichoic acid of Lactobacillus plantarum. Eur J Biochem 1985, 148(1):29-34.

38. Palumbo E, Deghorain M, Cocconcelli PS, Kleerebezem M, Geyer A, Hartung T, Morath S, Hols P: D-alanyl ester depletion of teichoic acids in Lactobacillus plantarum results in a major modification of lipoteichoic acid composition and cell wall perforations at the septum mediated by the Acm2 autolysin. J Bacteriol 2006, 188(10):3709-3715.

39. Honeyman AL, Stewart GC: The nucleotide sequence of the rodC operon of Bacillus subtilis. Mol Microbiol 1989, 3(9):1257-1268.

40. Xia G, Maier L, Sanchez-Carballo P, Li M, Otto M, Holst O, Peschel A: Glycosylation of wall teichoic acid in Staphylococcus aureus by TarM. J Biol Chem 2010, 285(18):13405-13415. 
41. Bron PA, Wels M, Bongers RS: van Bokhorst-van de Veen $M$, Wiersma $A$, Overmars L, Marco ML, Kleerebezem M: Transcriptomes reveal genetic signatures underlying physiological variations imposed by different fermentation conditions in Lactobacillus plantarum. PLOS ONE 2012, 7(7):e38720.

42. Vinogradov E, Sadovskaya I, Li J, Jabbouri S: Structural elucidation of the extracellular and cell-wall teichoic acids of Staphylococcus aureus MN8m, a biofilm forming strain. Carbohydr Res 2006, 341(6):738-743.

43. Dziarski R, Gupta D: Staphylococcus aureus peptidoglycan is a toll-like receptor 2 activator: a reevaluation. Infect Immun 2005, 73(8):5212-5216.

44. Travassos LH, Girardin SE, Philpott DJ, Blanot D, Nahori MA, Werts C, Boneca IG: Toll-like receptor 2-dependent bacterial sensing does not occur via peptidoglycan recognition. EMBO Rep 2004, 5(10):1000-1006.

45. Volz T, Nega M, Buschmann J, Kaesler S, Guenova E, Peschel A, Rocken M, Gotz F, Biedermann T: Natural Staphylococcus aureus-derived peptidoglycan fragments activate NOD2 and act as potent costimulators of the innate immune system exclusively in the presence of TLR signals. FASEB J 2010, 24(10):4089-4102.

46. Lebeer S, Vanderleyden J, De Keersmaecker SC: Genes and molecules of lactobacilli supporting probiotic action. Microbiol Mol Biol Rev 2008, 72(4):728-764. Table of Contents.

47. Han SH, Kim JH, Martin M, Michalek SM, Nahm MH: Pneumococcal lipoteichoic acid (LTA) is not as potent as staphylococcal LTA in stimulating Toll-like receptor 2. Infect Immun 2003, 71(10):5541-5548.

48. Kang JY, Nan X, Jin MS, Youn SJ, Ryu YH, Mah S, Han SH, Lee H, Paik SG, Lee JO: Recognition of lipopeptide patterns by Toll-like receptor 2-Toll-like receptor 6 heterodimer. Immunity 2009, 31(6):873-884.

49. Remus DM, Kleerebezem M, Bron PA: An intimate tete-a-tete - how probiotic lactobacilli communicate with the host. Eur J Pharmacol 2011, 668(Suppl 1):S33-S42.

50. Burian M, Rautenberg M, Kohler T, Fritz M, Krismer B, Unger C, Hoffmann WH, Peschel A, Wolz C, Goerke C: Temporal expression of adhesion factors and activity of global regulators during establishment of Staphylococcus aureus nasal colonization. J Infect Dis 2010, 201(9):1414-1421.

51. Marco ML, Peters TH, Bongers RS, Molenaar D, van Hemert S, Sonnenburg $J$, Gordon JI, Kleerebezem M: Lifestyle of Lactobacillus plantarum in the mouse caecum. Environ Microbiol 2009, 11(10):2747-2757.

52. Meijerink M, van Hemert $S$, Taverne $N$, Wels $M$, de Vos P, Bron PA, Savelkoul HF, van Bilsen J, Kleerebezem M, Wells JM: Identification of genetic loci in Lactobacillus plantarum that modulate the immune response of dendritic cells using comparative genome hybridization. PLoS One 2010, 5(5):e10632.

53. van Hemert S, Meijerink M, Molenaar D, Bron PA, de Vos P, Kleerebezem M, Wells JM, Marco ML: Identification of Lactobacillus plantarum genes modulating the cytokine response of human peripheral blood mononuclear cells. BMC Microbiol 2010, 10:293.

54. Teusink B, van Enckevort FH, Francke C, Wiersma A, Wegkamp A, Smid EJ, Siezen RJ: In silico reconstruction of the metabolic pathways of Lactobacillus plantarum: comparing predictions of nutrient requirements with those from growth experiments. App/ Environ Microbiol 2005, 71(11):7253-7262.

55. Casadaban MJ, Cohen SN: Analysis of gene control signals by DNA fusion and cloning in Escherichia coli. J Mol Biol 1980, 138(2):179-207.

56. Lambert JM, Bongers RS, Kleerebezem M: Cre-lox-based system for multiple gene deletions and selectable-marker removal in Lactobacillus plantarum. Appl Environ Microbiol 2007, 73(4):1126-1135.

57. Tomita S, Furihata K, Nukada T, Satoh E, Uchimura T, Okada S: Structures of two monomeric units of teichoic acid prepared from the cell wall of Lactobacillus plantarum NRIC 1068. Biosci Biotechnol Biochem 2009, 73(3):530-535.

58. Allen RJ: The estimation of phosphorus. Biochem J 1940, 34:858-865.

59. Dubois M, Gilles K, Hamilton JK, Rebers PA, Smith F: A colorimetric method for the determination of sugars. Nature 1951, 168(4265):167.

60. Morgan E, Varro R, Sepulveda H, Ember JA, Apgar J, Wilson J, Lowe L, Chen R, Shivraj L, Agadir A, et al: Cytometric bead array: a multiplexed assay platform with applications in various areas of biology. Clin Immunol 2004, 110(3):252-266.

61. Karczewski J, Troost FJ, Konings I, Dekker J, Kleerebezem M, Brummer RJ, Wells JM: Regulation of human epithelial tight junction proteins by
Lactobacillus plantarum in vivo and protective effects on the epithelial barrier. Am J Physiol Gastrointest Liver Physiol 2010, 298(6):G851-G859.

62. Pooley HM, Abellan FX, Karamata D: CDP-glycerol:poly(glycerophosphate) glycerophosphotransferase, which is involved in the synthesis of the major wall teichoic acid in Bacillus subtilis 168 , is encoded by tagF (rodC). J Bacteriol 1992, 174(2):646-649.

63. Meredith TC, Swoboda JG, Walker S: Late-stage polyribitol phosphate wall teichoic acid biosynthesis in Staphylococcus aureus. J Bacteriol 2008, 190(8):3046-3056

64. Lebeer S, Verhoeven TL, Perea Velez M, Vanderleyden J, De Keersmaecker SC: Impact of environmental and genetic factors on biofilm formation by the probiotic strain Lactobacillus rhamnosus GG. Appl Environ Microbiol 2007, 73(21):6768-6775.

65. Tabuchi Y, Shiratsuchi A, Kurokawa K, Gong JH, Sekimizu K, Lee BL, Nakanishi Y: Inhibitory role for D-alanylation of wall teichoic acid in activation of insect Toll pathway by peptidoglycan of Staphylococcus aureus. J Immunol 2010, 185(4):2424-2431.

66. Kerkhoven R, van Enckevort FH, Boekhorst J, Molenaar D, Siezen RJ: Visualization for genomics: the Microbial Genome Viewer. Bioinformatics 2004, 20(11):1812-1814.

67. Teusink B, Wiersma A, Molenaar D, Francke C, de Vos WM, Siezen RJ, Smid EJ: Analysis of growth of Lactobacillus plantarum WCFS1 on a complex medium using a genome-scale metabolic model. J Biol Chem 2006, 281(52):40041-40048.

doi:10.1186/1475-2859-11-123

Cite this article as: Bron et al: Lactobacillus plantarum possesses the capability for wall teichoic acid backbone alditol switching. Microbial Cell Factories 2012 11:123.

\section{Submit your next manuscript to BioMed Central and take full advantage of:}

- Convenient online submission

- Thorough peer review

- No space constraints or color figure charges

- Immediate publication on acceptance

- Inclusion in PubMed, CAS, Scopus and Google Scholar

- Research which is freely available for redistribution 